
"IF THIS BIA OBJEET IS OUT THERE, II BASICALLY GHANEES OUR PERGEPTION OF IHE SOLAR SYSTEM."
Astronomer Scott Sheppard is searching systematically for distant objects - one of which could be the elusive Planet $X$. seen in the Solar System.

Astronomers have discovered more than 2,000 exoplanets around other stars, mostly through indirect methods that detect changes in the distant star. Yet the farthest reaches of our Solar System remain largely unexplored: the indirect techniques won't work in our own stellar neighbourhood, and objects in the distant suburbs of the Sun are too faint to be glimpsed by anything but the world's most powerful telescopes. Sheppard and Trujillo have been racing to find the frigid worlds that are thought to populate this distant zone. At stake is what could be the last great discovery in the Solar System - a planet bigger than Earth that may swing around the Sun far past Pluto.

Suggestions of a Planet $\mathrm{X}$ have circulated for more than a century, but those hypotheses have always fallen apart after closer scrutiny. In 2014, Trujillo and Sheppard revived the concept of a putative Planet X, on the basis of the orbits of some extremely distant objects ${ }^{1}$. In January, the idea got a boost when two astronomers from the California Institute of Technology (Caltech) in Pasadena calculated more precisely where in the Solar System it might lie ${ }^{2}$. They dubbed it 'Planet Nine': a backhanded reference to the demotion of Pluto from planet to dwarf planet in 2006.

The hunt is now on to find Planet Nine, or any other unseen super-Earths that may lurk out there on other orbits. The quest is likely to reveal fundamental insights into how the Solar System formed 4.6 billion years ago, and how it has evolved since then. "If this big object is out there, it basically changes our perception of the Solar System," says Sheppard. "It's true discovery at its core."

\section{UNKNOWN ZONE}

When Sheppard and Trujillo began to chase distant worlds, they were hoping to follow in the footsteps of other legendary astronomers. In 1846, Johann Galle of Germany first spotted Neptune, the eighth planet, at a distance of about $30 \mathrm{AU}$, right where it was expected to be according to calculations of how it would gravitationally perturb Uranus. In 1930, US astronomer Clyde Tombaugh found Pluto, orbiting at a distance of around $40 \mathrm{AU}$. And in 1992, astronomers David Jewitt, then at the University of Hawaii, and Jane Luu, then at the University of California Berkeley, discovered an even more distant object, beginning the exploration of a region of space called the Kuiper belt ${ }^{3}$.

Since then, astronomers have found thousands of Kuiper belt objects: small icy worlds similar to Pluto that range in distance from about 30 $\mathrm{AU}$ to $50 \mathrm{AU}$ from the Sun. Beyond that lies the equivalent of 'here be dragons' on old maps. Scientists sometimes call it the outermost Kuiper belt or the innermost Oort cloud - the next region of the Solar System, which is thought to extend to at least 100,000 AU. "There's a whole chunk of the Solar System we don't fully understand," says Meg Schwamb, a planetary astronomer at the Academia Sinica in Taipei. "It's one of the last unexplored territories."

Which is why Sheppard and Trujillo are out looking. They met as graduate students at the University of Hawaii, where both had Jewitt as their adviser. They worked together to hunt Kuiper belt objects, and then started a systematic survey to search for still more-distant worlds. They are the only team routinely looking for the most-extreme objects. "The population could be huge," says Trujillo. "That's why we're doing the search."

By 2012, the two were using the biggest light buckets they could get their hands on, with wide-field cameras that would let them view as much of the sky as possible. At the Dark Energy Camera, atop a 4-metre telescope in Chile, they got a hit almost immediately. On their first night of observing, they spotted an object that was moving so slowly that it had to be very distant. Thrilled, they watched it move during the course of a year, which provided enough data to calculate its orbit.

They found that the closest it ever gets to the Sun - a measure known as its perihelion - is $80 \mathrm{AU}$, beyond the bulk of the Kuiper belt. This made it the object with the farthest-known perihelion, just beating the
Sun distance. It's not clear yet how big the object is or whether it is scientifically important - but it is among the most distant worlds ever 
dwarf planet Sedna, whose perihelion is $76 \mathrm{AU}$.

The discovery of this object, called $2012 \mathrm{VP}_{113}$, led to a Nature paper - and a lot more observing time on big telescopes. In 2014, Sheppard and Trujillo spent their first nights at Subaru, a facility run by the National Astronomical Observatory of Japan that carries a huge camera called the Hyper Suprime-Cam. The combination of a big telescope and a wide-field camera makes Subaru the world's best place to scan large sections of the sky for faint objects.

Many scientists work with Subaru remotely: they stay at sea level in Hilo, and use videoconferencing to communicate with the telescope's operators. That approach saves researchers from making the 2-hourlong journey to the summit of Mauna Kea at 4,200 metres, where the atmosphere has $40 \%$ less oxygen and causes many people to experience dizziness, headaches or sometimes more-serious medical problems.

But Sheppard likes to be actively involved in directing observations, so he always makes the trip. As the hours tick by during the night, he stays alert, never once clipping an oxygen sensor onto his finger to see how he is coping with the altitude. His logbook fills up with notations: field number, chip number, exposure time. He reorders targets on the fly, rearranging what he is looking at to improve the time gap between the fields.

Subaru's huge mirror gazes at the sky, gathering photons for him. Exposure times count down in big green numbers on a computer monitor. When an exposure finishes, an alert dings like a cuckoo clock, and Sheppard hovers over the shoulder of the telescope operator to tell him where to point the camera next.

Each good observing night fills up Sheppard's Macbook with data. To identify potential distant worlds, the researchers use a programme that Trujillo wrote to pick out objects that move between different frames of the same star field. But because the programme flags a number of false positives, each field must also be reviewed manually. Sheppard goes through every exposure, eyeballing the faint dots that the programme circled in orange to decide whether they represent a distant Solar System object or something else an asteroid or a cosmic-ray blip, perhaps.

Frame by frame, Sheppard zips through thousands of exposures as if he's playing a video game. "It's exciting to go through," he says. "Every image - you never know what you're going to get. It could be the image with the super-Earth in it."

The key is how slowly the objects move.

Asteroids are relatively close to Earth, and their position in the sky can shift by 30 arcseconds, or 0.008 degrees, each hour. Kuiper belt objects, which are much farther away, traverse about 3 arcseconds of sky each hour. Anything slower than that must be beyond the main Kuiper belt and is something that interests the search team.

Astronomers must observe an object multiple times over the course of a year to pin down its orbit and determine its perihelion. Just because an object is remote when it is discovered does not mean that it is scientifically important.

For instance, the object that Sheppard spotted at $90 \mathrm{AU}$ in November may have been at its closest approach to the Sun. If so, that would make it a record-breaker, situated beyond Sedna and $2012 \mathrm{VP}_{113}$. Or the object might be travelling on a path that takes it much, much closer to the Sun, perhaps $40 \mathrm{AU}$. That would make it less exciting, because its perihelion distance would place it squarely within the main Kuiper belt — meaning that it is just another ordinary Kuiper belt object, as opposed to one of the extreme worlds.

The same is true for an object that the scientists found at $103 \mathrm{AU}$ last November - the most distant ever observed. They will not know for many months whether that body stays in the outer Solar System, or whether it veers inward at its perihelion.

By far the most prized quarry out there is the hypothesized Planet Nine. In their 2014 Nature paper, Trujillo and Sheppard suggested — on the basis of the orbits of $2012 \mathrm{VP}_{113}$ and Sedna - that an unseen superEarth could lurk at roughly $250 \mathrm{AU}$. This January, Konstantin Batygin and Mike Brown of Caltech took these two bodies, along with four other distant Kuiper belt objects, and compared their orbits to narrow the calculations of where such a planet might lie.

All six objects share a common orbital property: when they pass closest to the Sun, they are travelling from north to south relative to the plane of the Solar System. If they had no relation to one another, they should not all share that orientation. A second line of argument is that the six objects are also physically clustered in space (see 'Out there') "They all point in the same direction and are all tilted at the same angle," says Batygin. "That's odd."

He and Brown argue that an unseen Planet Nine must be shepherding them into those clusters. It would be between 5 and 10 times the mass of Earth, and travel as close as $200 \mathrm{AU}$ to the Sun and as far away as 1,200 AU.

\section{FINDING THE NINTH}

Critics say that the argument rests on just a handful of weird Kuiper belt objects. "It's very small statistics," says David Nesvorný, a planetary scientist at the Southwest Research Institute in Boulder, Colorado, who nonetheless finds the concept intriguing. "It's as science should be - at the edge of believability."

Many astronomers are now running their own calculations to estimate the chances that Planet Nine exists in this particular orbit, and if not, where it might be. Samantha Lawler, of National Research Council-Herzberg in Victoria, Canada, is working with Nathan Kaib, of the University of Oklahoma in Norman, to explore how the presence of a super-Earth might affect the orbits of many Kuiper belt objects. Their preliminary results suggest that, if a Planet Nine were out there, it should have nudged the orbits of Kuiper belt objects in ways that do not reflect reality.

Planet Nine "is a cool idea, and it would be really neat if it was true", says Lawler. "But you have to be really careful."

Some answers may come from an ongoing project known as the Outer Solar System Origins Survey (OSSOS), run by a consortium of investigators. It is working to find and study all the observable Kuiper belt objects in a small patch of the sky in extraordinary detail - by following their orbits, classifying their colours and so on. That work has the potential to rule out the existence of Batygin and Brown's hypothesized Planet Nine - if OSSOS were to find a distant object in a region that should have been cleared out by the proposed planet.

Other astronomers have suggested alternative ways to hunt Planet Nine, such as looking at data from the Cassini spacecraft orbiting Saturn to see whether that planet's orbit is perturbed ever so slightly, or by using cosmological telescopes at the South Pole to detect a planet's faint radiation. As Sheppard and Trujillo continue their methodical survey of the sky, they are paying special attention to areas where Batygin and Brown say the planet could be. And the Caltech pair is chasing it as well, also using Subaru. "I'd be astonished if there isn't some kind of planet there," says Renu Malhotra, a theorist at the University of Arizona in Tucson. In a paper on the preprint server arXiv, she and her colleagues put forward a new analysis ${ }^{4}$ of where a super-Earth might lurk, on a different orbit from Batygin and Brown's Planet Nine. Malhotra's team uses four extreme Kuiper belt objects to suggest that an unseen planet moves around the Sun every 17,000 years.

But even if a large planet is out there, it will take some luck to find it with existing technology. For one of the teams to spot the object, it 


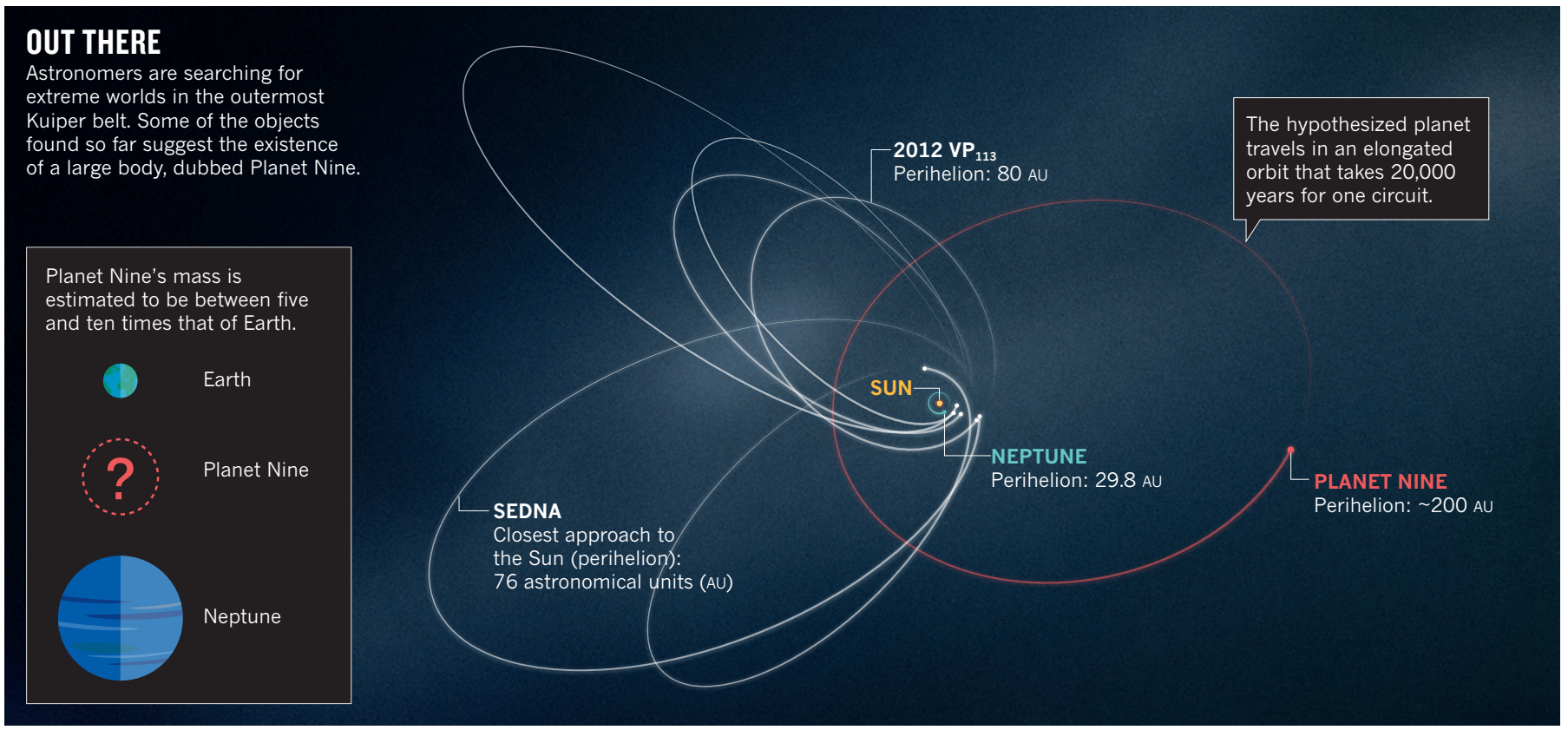

would have to be on the larger end of its estimated size range, or be very reflective or in a relatively close-in orbit. If the planet is too small, dark and far away, it may never be seen from Earth. "It's worse than looking for a needle in a haystack," says Malhotra. "It's like looking for the broken tip of a needle in a haystack."

\section{THE STORY OF A PLANET}

A more fundamental question is not whether Planet Nine exists, but what distant objects say about planetary evolution more generally. Discoveries such as Sedna and $2012 \mathrm{VP}_{113}$ have forced a radical rethinking of the gravitational forces that shape the outer parts of the Solar System.

When astronomers first started to find Kuiper belt objects in the 1990s and recognized that Pluto was just another member of that clan, they began to paint a picture of this mysterious realm of space. The Kuiper belt seemed to extend neatly from about $30 \mathrm{AU}$ to $50 \mathrm{AU}$, with most objects following stately orbits around the Sun. Those that were a bit odd - travelling off-kilter to the plane of the Solar System, or occasionally to greater distances - could be explained by gravitational interactions with Neptune.

Sedna and $2012 \mathrm{VP}_{113}$ do not fit that simple model because they range too far from the Sun to have ever interacted much with Neptune. Theorists suddenly had to confront the question of how these objects reached their current orbits. All known planets in the Solar System, along with the Kuiper belt objects, are thought to have condensed from a disk of gas and dust that swirled around the newborn Sun 4.6 billion years ago. But Sedna and other objects beyond the main Kuiper belt probably weren't born where they are today, because there simply wasn't enough gas and dust available at those great distances to create sizeable worlds.

One idea is that they were tossed there by a gravitational battle with other protoplanets closer to the Sun during the first tens of millions of years of the Solar System's existence. A second theory holds that the gravity of a passing star tugged on the outer bits of the planet-forming disk, pulling nascent planets into elongated orbits, where they remain today.

If Planet Nine exists, it could complicate this picture even more. It would mean that the orbits of Sedna and $2012 \mathrm{VP}_{113}$ were not fixed early on but are being actively shaped - even today — by the gravitational tugs of Planet Nine. That would require theorists to rewrite their ideas about how the Solar System's many worlds have interacted with one another over the past 4.6 billion years. "It's hard to anticipate what direction our imaginations will go," says Malhotra.

Understanding the distant Kuiper belt could also help astronomers to work out how our Solar System compares with planetary systems around other stars. Brown notes that one of the most common types of exoplanet is one missing from our Solar System, a world more massive than Earth but less massive than Neptune - that is, around the range of the hypothesized Planet Nine. "Maybe we can see what this most common type of planet might actually look like," he says.

For now, scientists' best shot at answering these questions is to find more extremely distant worlds. And that is why Sheppard and Trujillo keep plugging away in Chile and Hawaii, having covered less than 10\% of the sky that they intend to survey.

Back on Mauna Kea, Sheppard pushes through the night of observing, clocking one field after another with no break. By 4:45 a.m., the atmosphere above the summit is turning a little more opaque, and he begins to shift the exposure times longer. Finally, at 5:25 a.m., he turns to the videoconferencing unit and calls to his colleague in Hilo. "Chad, are you there?" he asks. "All the fields are in." The skies above Subaru are beginning to brighten, although Sheppard does not get to enjoy the spectacular view of a Hawaiian sunrise because he does not step outside. He is busy tallying his 33 fields for the night. Any one of them could contain a new extreme Kuiper belt object — or even a Planet Nine.

It is after 7 a.m. when the observing team tumbles into two sportsutility vehicles and drives the steep, rocky road down from Mauna Kea's summit. Sheppard starts to flag only when he sits down for breakfast at the astronomers' dorm, 1,360 metres lower down on the mountain. He and Tholen gulp down their food and retreat to black-curtained dormitories to sleep until noon.

Sheppard, aged 40, has asked his doctor about eye strain and whether he will be able to keep looking at star fields forever. He and Trujillo have a self-appointed goal of finding ten inner-Oort-cloud objects, a number that they think will enable them to start testing ideas for how these objects formed and evolved.

That means many more long nights at the telescope. "If it turns into postage-stamp collecting, we'll stop," Sheppard says. "But right now, every new discovery is a huge difference-maker in trying to understand what's going on out there."

Alexandra Witze writes for Nature from Boulder, Colorado. Not one to pull an all-nighter, she took a nap at Subaru.

1. Trujillo, C. A. \& Sheppard, S. S. Nature 507, 471-474 (2014)

2. Batygin, K. \& Brown, M. E. Astron. J. 151, 22 (2016).

3. Jewitt, D. \& Luu, J. Nature 362, 730-732 (1993)

4. Malhotra, R., Volk, K. \& Wang, X. Preprint at http://arxiv.org/abs/1603.02196v2 (2016). 